\title{
The Maximum Number of Triangles in a Graph of Given Maximum Degree
}

\author{
Zachary Chase*
}

Received 13 March 2020; Published 4 September 2020

\begin{abstract}
We prove that any graph on $n$ vertices with max degree $d$ has at most $q\left(\begin{array}{c}d+1 \\ 3\end{array}\right)+\left(\begin{array}{c}r \\ 3\end{array}\right)$ triangles, where $n=q(d+1)+r, 0 \leq r \leq d$. This resolves a conjecture of Gan-Loh-Sudakov.
\end{abstract}

\section{Introduction}

Fix positive integers $d$ and $n$ with $d+1 \leq n \leq 2 d+1$. Galvin [7] conjectured that the maximum number of cliques in an $n$-vertex graph with maximum degree $d$ comes from a disjoint union $K_{d+1} \sqcup K_{r}$ of a clique on $d+1$ vertices and a clique on $r:=n-d-1$ vertices. Cutler and Radcliffe [4] proved this conjecture. Engbers and Galvin [6] then conjectured that, for any fixed $t \geq 3$, the same graph $K_{d+1} \sqcup K_{r}$ maximizes the number of cliques of size $t$, over all $(d+1+r)$-vertex graphs with maximum degree $d$. Engbers and Galvin [6]; Alexander, Cutler, and Mink [1]; Law and McDiarmid [11]; and Alexander and Mink [2] all made progress on this conjecture before Gan, Loh, and Sudakov [9] resolved it in the affirmative. Gan, Loh, and Sudakov then extended the conjecture to arbitrary $n \geq 1$ (for any $d$ ).

Conjecture (Gan-Loh-Sudakov Conjecture). Any graph on $n$ vertices with maximum degree $d$ has at most $q\left(\begin{array}{c}d+1 \\ 3\end{array}\right)+\left(\begin{array}{l}r \\ 3\end{array}\right)$ triangles, where $n=q(d+1)+r, 0 \leq r \leq d$.

They showed their conjecture implies that, for any fixed $t \geq 4$, any max-degree $d$ graph on $n=$ $q(d+1)+r$ vertices has at most $q\left(\begin{array}{c}d+1 \\ t\end{array}\right)+\left(\begin{array}{c}r \\ t\end{array}\right)$ cliques of size $t$. In other words, considering triangles is enough to resolve the general problem of cliques of any fixed size.

\footnotetext{
*The author is partially supported by Ben Green's Simons Investigator Grant 376201 and gratefully acknowledges the support of the Simons Foundation.
} 


\section{ZACHARY CHASE}

The Gan-Loh-Sudakov conjecture (GLS conjecture) has attracted substantial attention. Cutler and Radcliffe [5] proved the conjecture for $d \leq 6$ and showed that a minimal counterexample, in terms of number of vertices, must have $q=O(d)$. Gan [8] proved the conjecture if $d+1-\frac{9}{4096} d \leq r \leq d$ (there are some errors in his proof, but they can be mended). Using fourier analysis, the author [3] proved the conjecture for Cayley graphs with $q \geq 7$. Kirsch and Radcliffe [10] investigated a variant of the GLS conjecture in which the number of edges is fixed instead of the number of vertices (with still a maximum degree condition).

In this paper, we fully resolve the Gan-Loh-Sudakov conjecture.

Theorem 1. For any positive integers $n, d \geq 1$, any graph on $n$ vertices with maximum degree $d$ has at most $q\left(\begin{array}{c}d+1 \\ 3\end{array}\right)+\left(\begin{array}{l}r \\ 3\end{array}\right)$ triangles, where $n=q(d+1)+r, 0 \leq r \leq d$.

Analyzing the proof shows that $q K_{d+1} \sqcup K_{r}$ is the unique extremal graph if $r \geq 3$, and that $q K_{d+1} \sqcup H$, for any $H$ on $r$ vertices, are the extremal graphs if $0 \leq r \leq 2$.

The heart of the proof is the following Lemma, of independent interest, which says that, in any graph, we can find a closed neighborhood whose removal from the graph removes few triangles. Theorem 1 will follow from its repeated application.

Lemma 1. In any graph $G$, there is a vertex $v$ whose closed neighborhood meets at most $\left(\begin{array}{c}d(v)+1 \\ 3\end{array}\right)$ triangles.

As mentioned above, Theorem 1, together with the work of Gan, Loh, and Sudakov [9], yields the general result, for cliques of any fixed size.

Theorem 2. Fix $t \geq 3$. For any positive integers $n, d \geq 1$, any graph on $n$ vertices with maximum degree $d$ has at most $q\left(\begin{array}{c}d+1 \\ t\end{array}\right)+\left(\begin{array}{c}r \\ t\end{array}\right)$ cliques of size $t$, where $n=q(d+1)+r, 0 \leq r \leq d$.

Theorem 2 gives another proof of (the generalization of) Galvin's conjecture (to $n \geq 2 d+2$ ) that a disjoint union of cliques maximizes the total number of cliques in a graph with prescribed number of vertices and maximum degree.

Finally, the author would like to point out a connection to a related problem, that of determining the minimum number of triangles that a graph of fixed number of vertices $n$ and prescribed minimum degree $\delta$ can have. The connection stems from a relation, observed in [2] and [9], between the number of triangles in a graph and the number of triangles in its complement:

$$
|T(G)|+\left|T\left(G^{c}\right)\right|=\left(\begin{array}{l}
n \\
3
\end{array}\right)-\frac{1}{2} \sum_{v} d(v)[n-1-d(v)] .
$$

Lo [12] resolved this "dual" problem when $\delta \leq \frac{4 n}{5}$. His results resolve the GLS conjecture for regular graphs for $q=2,3$, and the GLS conjecture implies his results, up to an additive factor of $O\left(\delta^{2}\right)$, for $q=2,3$, and yields an extension of his results for $q \geq 4-$ these are the optimal results asymptotically, in the natural regime of $\frac{\delta}{n}$ fixed, and $n \rightarrow \infty$. 
The Maximum Number of Triangles in a Graph of Given Maximum Degree

\section{Notation}

Denote by $E$ the edge set of $G$; for two vertices $u, v$, we write " $u v \in E$ " if there is an edge between $u$ and $v$ and " $u v \notin E$ " otherwise — in particular, for any $u, u u \notin E$. For a vertex $v$, let $\left|T_{N[v]}\right|$ denote the number of triangles with at least one vertex in the closed neighborhood $N[v]:=\{u: u v \in E\} \cup\{v\}$, and let $|T(G-N[v])|$ denote the number of triangles with all vertices in the graph $G-N[v]$ (the subgraph induced by the vertices not in $N[v])$. Finally, $d(v)$ denotes the degree of $v$.

\section{Proof of Theorem 1}

For a graph $G$, let $W(G)=\{(x, u, v, w): u x, v x, w x \in E, u v, u w, v w \notin E\}$.

Lemma 2. For any graph $G, 6 \sum_{v}\left|T_{N[v]}\right|+|W(G)|=\sum_{v} d(v)^{3}$.

Proof. Let $\Omega=\{(z, u, v, w): u v, u w, v w \in E$ and $[z u \in E$ or $z v \in E$ or $z w \in E]\}, \Sigma=\{(x, u, v, w): u x, v x, w x \in$ $E\}$, and $W=W(G)$. Note that repeated vertices in the 4-tuples are allowed. First observe that, since there are 6 ways to order the vertices of a triangle, $\sum_{v} 6\left|T_{N[v]}\right|=|\Omega|$. Any 4-tuple in $\Sigma, W$, or $\Omega$ gives rise to one of the induced subgraphs shown below, since one vertex must be adjacent to all the others.

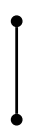

A

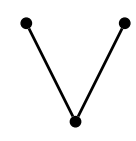

$B$

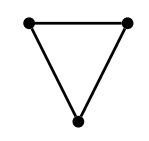

$C$

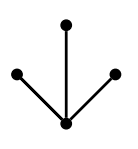

$D$

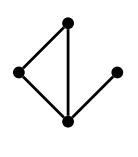

$F$

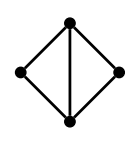

$H$

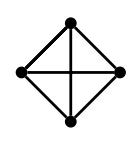

$I$

Since $|\Sigma|=\sum_{v} d(v)^{3}$, it thus suffices to show that for each of the induced subgraphs above, the number of times it comes from a 4-tuple in $\Sigma$ is the sum of the number of times it comes from 4-tuples in $\Omega$ and $W$. Any fixed copy of $A$, say on vertices $u$ and $v$, comes 0 times from a 4-tuple in $\Omega$ (since it has no triangles), and 2 times from each of $W$ and $\Sigma((u, v, v, v),(v, u, u, u))$. Any fixed copy of $B$, say on vertices $u, v, w$ with $v u, v w \in E$, comes 0 times from $\Omega$, and 6 times from each of $W$ and $\Sigma$ $((v, u, u, w),(v, u, w, u),(v, u, w, w),(v, w, u, u),(v, w, u, w),(v, w, w, u))$. Any fixed copy of $C$ comes 18 times from each of $\Omega$ and $\Sigma$ ( 3 choices for the first vertex and then 6 for the ordered triangle), and 0 times from $W$. Similarly, any fixed copy of $D$ comes 6 times from each of $W$ and $\Sigma$, and 0 times from $\Omega$; finally, $F, H, I$ come 6,12,24 times, respectively, from each of $\Omega$ and $\Sigma$, and 0 times from $W$.

We now prove our key lemma, previously mentioned in the introduction.

Lemma 1. In any graph $G$, there is a vertex $v$ whose closed neighborhood meets at most $\left(\begin{array}{c}d(v)+1 \\ 3\end{array}\right)$ triangles, i.e. $\left|T_{N[v]}\right| \leq\left(\begin{array}{c}d(v)+1 \\ 3\end{array}\right)$.

Proof. By Lemma 2, since $|W(G)| \geq|\{(x, u, u, u): u x \in E\}|=\sum_{x} d(x)$, we have $\sum_{v}\left|T_{N[v]}\right| \leq \sum_{v} \frac{1}{6}\left[d(v)^{3}-\right.$ $d(v)$. By the pigeonhole principle, there is some $v$ with

$$
\left|T_{N[v]}\right| \leq \frac{1}{6}\left[d(v)^{3}-d(v)\right]=\left(\begin{array}{c}
d(v)+1 \\
3
\end{array}\right) .
$$




\section{ZACHARY CHASE}

Lemma 3. For any positive integers $a \geq b \geq 1$, it holds that $\left(\begin{array}{l}a \\ 3\end{array}\right)+\left(\begin{array}{c}b \\ 3\end{array}\right) \leq\left(\begin{array}{c}a+1 \\ 3\end{array}\right)+\left(\begin{array}{c}b-1 \\ 3\end{array}\right)$. Consequently, for any positive integers $a, b$ and any positive integer $c$ with $\max (a, b) \leq c \leq a+b$, it holds that $\left(\begin{array}{l}a \\ 3\end{array}\right)+\left(\begin{array}{l}b \\ 3\end{array}\right) \leq$ $\left(\begin{array}{l}c \\ 3\end{array}\right)+\left(\begin{array}{c}a+b-c \\ 3\end{array}\right)$.

Proof. $\left(\begin{array}{c}a+1 \\ 3\end{array}\right)-\left(\begin{array}{l}a \\ 3\end{array}\right)=\left(\begin{array}{l}a \\ 2\end{array}\right)$, and $\left(\begin{array}{l}b \\ 3\end{array}\right)-\left(\begin{array}{c}b-1 \\ 3\end{array}\right)=\left(\begin{array}{c}b-1 \\ 2\end{array}\right)$. Iterate to get the consequence.

We now finish the proof of Theorem 1 .

Proof of Theorem 1. With a fixed $d$, we induct on $n$. For $n=1$, the result is obvious. Take some $n \geq 2$, and suppose the theorem holds for all smaller values of $n$. Let $G$ be a max-degree $d$ graph on $n$ vertices. By Lemma 1, we may take $v$ with $\left|T_{N[v]}\right| \leq\left(\begin{array}{c}d(v)+1 \\ 3\end{array}\right)$. Write $n=q(d+1)+r$ for $0 \leq r \leq d$. Note $|T(G)|=|T(G-N[v])|+\left|T_{N[v]}\right|$. Since $G-N[v]$ has maximum degree (at most) $d$, if $d(v)+1 \leq r$, then induction and Lemma 3 give

$$
|T(G)| \leq q\left(\begin{array}{c}
d+1 \\
3
\end{array}\right)+\left(\begin{array}{c}
r-(d(v)+1) \\
3
\end{array}\right)+\left(\begin{array}{c}
d(v)+1 \\
3
\end{array}\right) \leq q\left(\begin{array}{c}
d+1 \\
3
\end{array}\right)+\left(\begin{array}{l}
r \\
3
\end{array}\right)
$$

and if $d(v)+1>r$, then induction and Lemma 3 give

$$
|T(G)| \leq(q-1)\left(\begin{array}{c}
d+1 \\
3
\end{array}\right)+\left(\begin{array}{c}
d+1+r-(d(v)+1) \\
3
\end{array}\right)+\left(\begin{array}{c}
d(v)+1 \\
3
\end{array}\right) \leq q\left(\begin{array}{c}
d+1 \\
3
\end{array}\right)+\left(\begin{array}{l}
r \\
3
\end{array}\right) .
$$

The maximum degree condition ensured $d+1+r-(d(v)+1) \geq 0$ and $d(v)+1 \leq d+1$.

\section{Acknowledgments}

I would like to thank Po-Shen Loh for telling me the Gan-Loh-Sudakov conjecture and my advisor Ben Green for encouragement. I also thank Daniel Korandi for a cleaner proof of Lemma 2 and for helpful suggestions on the paper's presentation.

\section{References}

[1] J. Alexander, J. Cutler, and T. Mink, Independent Sets in Graphs with Given Minimum Degree, Electr. J. Comb. 19 (2012), 37.

[2] J. Alexander and T. Mink, A new method for enumerating independent sets of a fixed size in general graphs, J. Graph Theory 81 (2016), no. 1, 57-72.

[3] Z. Chase, The Maximum Number of Three Term Arithmetic Progressions, and Triangles in Cayley Graphs, preprint, ArXiv:1809.03729, 2018

[4] J. Cutler and A. J. Radcliffe, The maximum number of complete subgraphs in a graph with given maximum degree, J. Combin. Theory Ser. B 104 (2014), 60-71. 
[5] J. Cutler and A.J. Radcliffe, The maximum number of complete subgraphs of fixed size in a graph with given maximum degree, Journal of Graph Theory, 84(2):134-145, 2017.

[6] J. Engbers and D. Galvin, Counting independent sets of a fixed size in graphs with a given minimum degree, Journal of Graph Theory 76(2) (2014), 149-168.

[7] D. Galvin, Two problems on independent sets in graphs, Discrete Math. 311 (2011), no. 20, 2105 2112.

[8] W. Gan, Several Problems in Extremal Combinatorics, 2014, PhD Thesis.

[9] W. Gan, P. Loh, and B. Sudakov, Maximizing the number of independent sets of a fixed size, Combinatorics, Probability and Computing 24 (2015), 521-527.

[10] R. Kirsch and A. J. Radcliffe, Many triangles with few edges, Electr. J. Combin., 26(2):Paper 2.36, 23, 2019.

[11] H. Law and C. McDiarmid, On Independent Sets in Graphs with Given Minimum Degree, Combinatorics, Probability and Computing 22 (2013), no. 6, 874-884.

[12] A. Lo, Cliques in graphs with bounded minimum degree, Combin. Probab. Comput. 21 (2012), 457-482.

\section{AUTHOR}

Zachary Chase

University of Oxford

Oxford, United Kingdom

zachary.chase@maths.ox.uk.edu

http://people.maths.ox.ac.uk/ chase/ 